\title{
Targeting a novel domain in podoplanin for inhibiting platelet- mediated tumor metastasis
}

\author{
Takaya Sekiguchi ${ }^{1,2}$, Ai Takemoto ${ }^{1}$, Satoshi Takagi ${ }^{1}$, Kazuki Takatori, ${ }^{1,2}$, Shigeo Sato ${ }^{1}$, \\ Miho Takami ${ }^{1}$, Naoya Fujita ${ }^{1,2}$ \\ ${ }^{1}$ Division of Experimental Chemotherapy, Cancer Chemotherapy Center, Japanese Foundation for Cancer Research, Tokyo, \\ Japan \\ ${ }^{2}$ Department of Computational Biology and Medical Sciences, Graduate School of Frontier Sciences, The University of Tokyo, \\ Japan
}

Correspondence to: Naoya Fujita, e-mail: naoya.fujita@jfcr.or.jp

Keywords: podoplanin, platelets, platelet aggregation, neutralizing antibody, tumor metastasis

Received: August 28, 2015

Accepted: November 22, 2015

Published: December 14, 2015

\section{ABSTRACT}

Podoplanin/Aggrus is a sialoglycoprotein expressed in various cancers. We previously identified podoplanin as a key factor in tumor-induced platelet aggregation. Podoplanin-mediated platelet aggregation enhances tumor growth and metastasis by secreting growth factors and by forming tumor emboli in the microvasculature. Thus, precise analysis of the mechanisms of podoplanin-mediated platelet aggregation is critical for developing anti-tumor therapies. Here we report the discovery of a novel platelet aggregation-inducing domain, PLAG4 (81-EDLPT-85). PLAG4 has high homology to the previously reported PLAG3 and contributes to the binding of its platelet receptor CLEC-2. Mutant analyses indicated that PLAG4 exhibits a predominant platelet-aggregating function relative to PLAG3 and that conserved Glu ${ }^{81} /$ Asp $^{82} /$ Thr $^{85}$ residues in PLAG4 are indispensable for CLEC-2 binding. By establishing anti-PLAG4neutralizing monoclonal antibodies, we confirmed its role in CLEC-2 binding, platelet aggregation, and tumor emboli formation. Our results suggest the requirement of simultaneous inhibition of PLAG3/4 for complete suppression of podoplanin-mediated tumor growth and metastasis.

\section{INTRODUCTION}

Tumor cell growth and survival are affected by a wide variety of tumor microenvironments. During hematogenous metastasis processes, circulating tumor cells rarely survive due to exclusion by the immune system. Platelets are known as the critical component that affects the survival of circulating tumor cells, leading to metastasis formation [1]. In fact, anti-platelet agents and thrombocytopenia reduce tumor metastasis in experimental models $[2,3]$. Moreover, the administration of anti-coagulants has been reported to lower the mortality rate $[4,5]$. The following mechanisms underlying plateletpromoting metastasis are proposed: (i) enhancement of tumor cell embolization in the microvasculature by the formation of large tumor cell-platelet aggregates, (ii) up-regulation of tumor malignancy by the release of soluble factors from activated platelets, and (iii) protection from immunological assault or blood shear stress by coating of the tumor cell surface [1]. As all pathways are triggered by tumor-platelet interaction, this interaction could be a promising target for cancer therapy.

We have previously identified a type-I transmembrane sialoglycoprotein, podoplanin, also known as Aggrus, as a platelet aggregation-inducing factor in highly metastatic tumor cells [6]. Podoplanin expression induces platelet aggregation, and experimental and spontaneous pulmonary metastasis [3, 6]. Podoplanin is frequently overexpressed in various malignant tumors such as squamous cell carcinoma, mesothelioma, glioblastoma, bladder tumors, and osteosarcoma [7-11]. Therefore, the platelet-interaction and the platelet-aggregating ability of podoplanin may be a target for suppressing metastasis in clinical situations.

Podoplanin contains three tandemly repeated EDXXVTPG motifs in the extracellular domain. Analysis of the epitope of a neutralizing anti-mouse podoplanin monoclonal antibody $(\mathrm{mAb}) 8 \mathrm{~F} 11$ helped to identify the domains critical for exhibiting platelet-aggregating ability. Therefore, we designated the motif-containing domain (EDXXVTPG; in which " $\mathrm{X}$ " may be any amino acid) as 
the PLatelet AGgregation-stimulating (PLAG) domain (PLAG1-3) [6]. These domains are highly conserved among mammals in a triplicated manner. The PLAG1 and/or PLAG3 domain contains a predicted glycosylated Thr residue that is critical for podoplanin activity $[3,6,12]$. $O$-glycanase treatment reduces podoplanin's platelet aggregation ability [13], and studies using a series of glycosylation-deficient $\mathrm{CHO}$ cell mutants and genetically modified yeast suggest the requirement of sialylated $O$-glycan for podoplanin-induced platelet aggregation $[14,15]$. In fact, a disialyl-core structure on $\mathrm{Thr}^{52}$ in PLAG3 has been detected in human podoplanin [16].

The C-type lectin-like receptor 2 (CLEC-2), originally identified as a platelet receptor for the snake venom toxin rhodocytin [17], has been identified as a podoplanin receptor [18]. Podoplanin binding to CLEC-2 transmits platelet-activation signals through Src family kinases, Syk, and phospholipase $\mathrm{C} \gamma 2$ in platelets $[17,19]$. CLEC-2-deficient platelets can still respond to platelet agonists, such as collagen and ADP, suggesting the possibility that the interfering podoplanin-CLEC-2 interaction may not affect physiological hemostasis [20]. Till date, we and another group have generated several PLAG domain-recognizing anti-human podoplanin mAbs that suppress podoplanin binding to CLEC-2, platelet aggregation, and hematogenous metastasis [21-23]. Because our generated anti-podoplanin neutralizing mAbs, P2-0 and MS-1, recognized Gly ${ }^{45}$ in PLAG2 and $\mathrm{Asp}^{48}$ and $\mathrm{Asp}^{49}$ in PLAG3, the perimeter structure around PLAG2 and PLAG3 could be the CLEC-2-binding site $[22,23]$. A recent report on the crystal structure of human podoplanin-derived $O$-glycosylated PLAG2-PLAG3 peptide in complex with CLEC-2 extracellular domain revealed that the acidic side chains of $\mathrm{Glu}^{47}, \mathrm{Asp}^{48}$, and the sialic acid attached to $\mathrm{Thr}^{52}$ on PLAG3 were recognized by basic residues on CLEC-2 [24].

Here we report the discovery of an additional PLAG domain (81-85 aa in human podoplanin), which is highly conserved among mammals. The region includes the highly conserved EDXXT motif, which is closely related to the PLAG domain consensus sequence EDXXVTPG. Deletion of the region or a point mutation in it drastically attenuated its binding to CLEC-2 and podoplanin-induced platelet aggregation. Thus, we designated the region as the fourth PLAG domain, PLAG4. Importantly, deletions or point mutations in both PLAG3 and PLAG4 domains almost completely suppressed the CLEC-2-binding ability and platelet-aggregating activity of human podoplanin, whereas the deletion of or point mutation in either one of the two domains could not completely suppress the binding ability and platelet-aggregating ability. To further investigate the requirement of the PLAG4 domain for podoplanin's platelet-aggregating ability, we tried to establish PLAG4recognizing mAbs. The generated PLAG4-recognizing mAbs exhibited CLEC-2-binding inhibitory activity and platelet aggregation-neutralizing ability. Moreover, the injection of the mAbs suppressed podoplanin-mediated hematogenous metastasis in vivo. These results suggest that the newly identified PLAG4 domain plays a critical role in the binding to CLEC-2, hence being a promising target motif for suppressing podoplanin-expressing tumor cell growth and metastasis.

\section{RESULTS}

\section{Identification of a novel CLEC-2-binding domain on human podoplanin}

We previously identified that podoplanin contains three tandemly repeated PLAG domains (PLAG1-3, Figure 1A) that are critical to its platelet aggregationinducing ability [6]. In addition, glycosylations on podoplanin are reported to be essential for its platelet aggregation-inducing ability $[14,15]$. To evaluate their contributions to CLEC-2 binding, we estimated the recombinant CLEC-2 binding to $\mathrm{CHO}$ cells that had been transfected with a PLAG1- or PLAG3-deleted podoplanin cDNA-containing plasmid. We generated a PLAG1-deletion mutant by deleting the 29-34 aa-coding region and a PLAG3-deletion mutant by deleting the 47-52 aa-coding region (Figure $1 \mathrm{~B}$ ). We confirmed that the expression level of wild type (WT) or deleted podoplanin was almost the same among the transfectants (Figure 1C, left panels). Surprisingly, the $\Delta 29-34 /$ PLAG1 deletion did not affect the binding of podoplanin to CLEC-2 (Figure 1C, right panels). Interestingly, the deletion of $\triangle 47-52 /$ PLAG3 could not abrogate podoplanin binding to CLEC-2 but only showed a partial reduction of its binding capability (Figure 1C, right panels). These results suggest that other regions in podoplanin may be associated with the binding to CLEC-2. We therefore analyzed the highly conserved regions of mammalian podoplanin amino acid sequences (Figure 1D). Sequences of 42 mammalian species retrieved from the NCBI Reference Sequence Database were selected (Supplementary Figure S1), and data were analyzed using sliding-window analysis and hydropathy plots (Figure 1D). Apart from the N-terminal signal peptide, we found four highly conserved regions within the extracellular domain (red dotted lines in Figure 1D). Three out of four regions contained highly negativecharged motifs, and the forth conserved region did not (hydropathy plots in Figure 1D). We studied them in detail and found that the three acidic regions were composed of two negatively charged amino acids followed by a Thr residue (Figure 1E) and that the forth region contained a completely different conserved sequence TSHS (106-109 aa). Consequently, the first region was identified as the PLAG1 domain, the second region was located in the PLAG3 domain, and the third region was located in the middle region (81-85 aa). Because no analysis of the third region had been carried out thus far, we further analyzed 


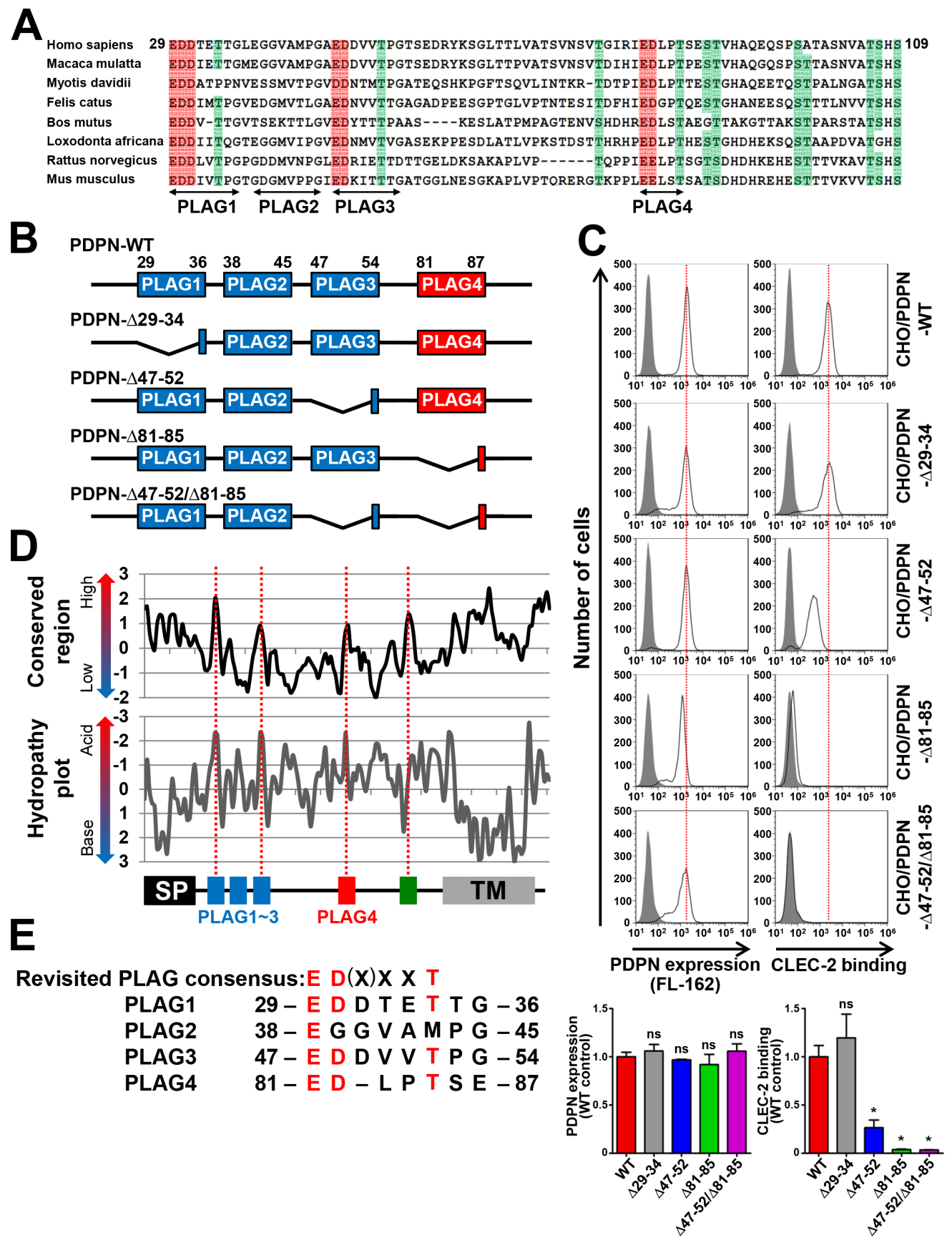

Figure 1: Identification of a new CLEC-2-binding domain, PLAG4, highly conserved in mammals. (A) Eight mammalian podoplanin protein sequences were aligned. Half-tone meshing area indicates over $80 \%$ conserved residues (Red: Asp or Glu, Green: Thr or Ser). The gaps against the Homo sapiens podoplanin found in multi-aligned sequences were deleted to show the alignment concisely. The deleted gaps in each sequence of species are followed. Macaca mulatta (XP_001106933.2), not deleted; Myotis davidii (XP_006766770.1), $\Delta$ A129; Bos mutus (XP_005889851.1), $\Delta$ P100-P112; Felis catus (XP_006934362.1), $\Delta$ T63; Loxodonta africana (XP_010591406.1), $\Delta \mathrm{T} 63$ and $\Delta \mathrm{H} 92$; Rattus norvegicus (NP_062231.1), $\Delta \mathrm{T} 63$; and Mus musculus (NP_034459.2), $\Delta \mathrm{T} 63$. (B) Schematic representation of human PLAG domain-deleted mutants used in this study. (C) CHO cells that had been stably transfected with PDPNWT or PLAG domain-deleted PDPN mutants were treated with control rabbit IgG (closed areas) or anti-PDPN pAb (FL162; open areas) for measuring PDPN expression levels (upper left panels), or with PBS (closed areas) or CLEC-2-(His) ${ }_{10}$ (open areas) for estimating CLEC-2-binding ability (upper right panels). After washing, cells were incubated with Alexa Flour 488-conjugated second antibody. The flow cytometry data (upper) and their quantitative graphs (lower) are shown. Each value in the lower graphs indicates mean \pm SDs $(N=3)$ of the peak values normalized by that of PDPN-WT/CHO. ${ }^{*} P<0.05$ using Mann-Whitney $U$ test. ns, not significant. (D) Sliding-window analysis and hydropathy analysis were performed using data from 42 mammalian species (window size equals three or two amino acids, respectively). Four highly conserved regions within the extracellular domains are indicated by red dotted lines. (E) Human PLAG1-4 domains were aligned with the revised PLAG consensus sequence ED(X)XXT. 
its role in CLEC-2 binding and platelet aggregation. We established $\mathrm{CHO}$ cells that had been transfected with $\Delta 81-85$-podoplanin and examined its ability to bind to CLEC-2 (Figure 1B and 1C). Surprisingly, the deletion of 81-85 aa attenuated the CLEC-2-binding ability more than the $\triangle 47-52 /$ PLAG3 deletion, and the double deletion of $\Delta 47-52 /$ PLAG3 and $\Delta 81-85$ almost completely suppressed the binding capability. Deletion of $81-85$ aa residues did not affect the membrane localization or expression level (Figure 1C). Thus, we speculated that this locus was associated with CLEC-2 binding, similarly to our previously reported PLAG domain. We therefore designated the region as the "PLAG4" domain (Figure 1E).

\section{Critical roles of PLAG4 domain in CLEC-2 binding and platelet aggregation}

To exclude the possibility that the deletion of domains affected 3D conformation, potentially leading to changes in the CLEC-2 interaction surface, we tried to generate human podoplanin point mutants exhibiting low affinity to CLEC-2. Our previously established neutralizing anti-human podoplanin $\mathrm{mAbs}, \mathrm{P} 2-0$ and MS-1, interfere with the binding of human podoplanin to CLEC-2, recognizing the perimeter structure around $\mathrm{Gly}^{45}, \mathrm{Asp}^{48}$, and $\mathrm{Asp}^{49}$ residues over PLAG2 and PLAG3 domains in human podoplanin $[22,23]$ (Supplementary Figure S2A). Because the $\mathrm{Asp}^{48}$ residue is an amino acid that is critical for the recognition by anti-PLAG3 mAbs (Supplementary Figure S2A) and its acidic side chain is suggested for the binding to CLEC-2 [24], we expected that the highly conserved $\mathrm{Asp}^{48}$ residue in the PLAG3 domain would play an important role in the interaction with CLEC-2. In fact, the substitution of $\mathrm{Asp}^{48}$ residue in PLAG3 to Ala (D48A) reduced the CLEC-2 binding, and compared with the D48A, the substitution of the $\mathrm{Asp}^{82}$ residue in PLAG4 to Ala (D82A) partially but rather significantly reduced the binding to CLEC-2 (Figure 2A and 2B). Consistently with those Asp mutations, either mutation of $\mathrm{Glu}^{47}$ or $\mathrm{Thr}^{52}$ in PLAG3, and $\mathrm{Glu}^{81}$ or $\mathrm{Thr}^{85}$ in PLAG4 showed the reduction in the CLEC-2 binding (Supplementary Figure S2B). Thus, those three, Glu, Asp, and Thr are critical conserved residues for CLEC-2 binding in both PLAG domains. Importantly, the double mutant harboring D48A and D82A mutations almost completely lost the affinity to CLEC-2 (Figure 2B). These results were consistent with the results obtained using PLAG-deletion mutants (Figure 1C). Consistent with CLEC-2-binding activities, single mutations at $\mathrm{Asp}^{48}$ or $\mathrm{Asp}^{82}$ (D48A or D82A) also lowered the plateletaggregating ability when compared with WT podoplanin (Figure 2C). Moreover, double mutant D48A/D82A could no longer exhibit platelet-aggregating ability (Figure $2 \mathrm{C}$ ). These results indicate that PLAG4 plays a crucial role in podoplanin-induced platelet aggregation via the formation of a complex with CLEC-2.

\section{Establishment and characterization of anti-PLAG4 monoclonal antibodies}

To further estimate the role of the PLAG4 domain in podoplanin-induced platelet aggregation and in pulmonary metastasis promoted by platelet aggregation, we attempted to establish a mouse $\mathrm{mAb}$ recognizing the PLAG4 domain. We purified a 12- or 40-times tandemly repeated human podoplanin PLAG4 peptide (TGIRIEDLPTSEST; 76-89 aa) as an antigen from a bacteria lysate and immunized the mice with the peptide. After fusing splenocytes with P3U1 myeloma cells, we screened hybridomas that secreted mAbs recognizing the immunogen, using enzyme-linked immunosorbent assay (ELISA)-based competition analysis. Upon further selection by recognition capability for podoplaninexpressing CHO/PDPN cells, but not for $\mathrm{CHO} /$ mock cells, we finally obtained two anti-PLAG4 mAbs, designated PG4D1 and PG4D2. The epitopes of PG4D1 and PG4D2 were identified by examining their reactivity to recombinant human $\Delta \mathrm{N} 24$-podoplanin expressed in $E$. coli harboring point mutations of each amino acid to Ala (Ala scanning; Figure 3A). We confirmed that PG4D1 and PG4D2 mAbs recognized the perimeter structure from $\mathrm{Arg}^{79}$ to $\mathrm{Leu}^{83}$ (Figure 3A). Each amino acid from $\mathrm{Arg}^{79}$ to $\mathrm{Leu}^{83}$ was required for PG4D1 mAb recognition of human podoplanin. Within the recognition epitope, $\mathrm{Arg}^{79}, \mathrm{Ile}^{80}, \mathrm{Glu}^{81}$, and $\mathrm{Leu}^{83}$ residues were required for PG4D2 recognition to podoplanin, whereas the Asp ${ }^{82}$ residue appeared to be less effective than other amino acids because PG4D2 mAb could weakly recognize D82A mutant (Figure 3A and Supplementary Figure S3A). We confirmed that both mAbs specifically recognized the PLAG4 domain using PLAG deletion mutants (Figure 3B). The subclasses of PG4D1 and PG4D2 mAbs were identified to be $\operatorname{IgG} 1$ and $\operatorname{IgG} 2 \mathrm{a}$, respectively (data not shown). Surface plasmon resonance (SPR) analysis revealed that both $\mathrm{mAbs}$ exhibited a high affinity to human podoplanin (Figure 3C). The dissociation rate constant $\left(k_{\mathrm{d}}\right)$ of both mAbs failed to reach values measurable by Biacore X100 due to the low level of dissociation, and so the precise equilibrium dissociation constant $\left(K_{\mathrm{D}}\right)$ values could not be determined. However, the $K_{\mathrm{D}}$ values of both mAbs were less than $0.3 \mathrm{nM}$.

\section{Anti-PLAG4 mAbs PG4D1 and PG4D2 exhibit inhibitory activity against podoplanin-CLEC-2 binding, platelet aggregation, and pulmonary metastasis in vivo}

To investigate the inhibitory activity of antiPLAG4 mAbs against podoplanin-CLEC-2 interaction, we performed alpha screen-based competition analysis using recombinant human Podoplanin-Fc and CLEC-2-(His) ${ }_{10}$ proteins. The addition of each $\mathrm{mAb}$ to the reactants suppressed podoplanin-CLEC-2 interaction in a 

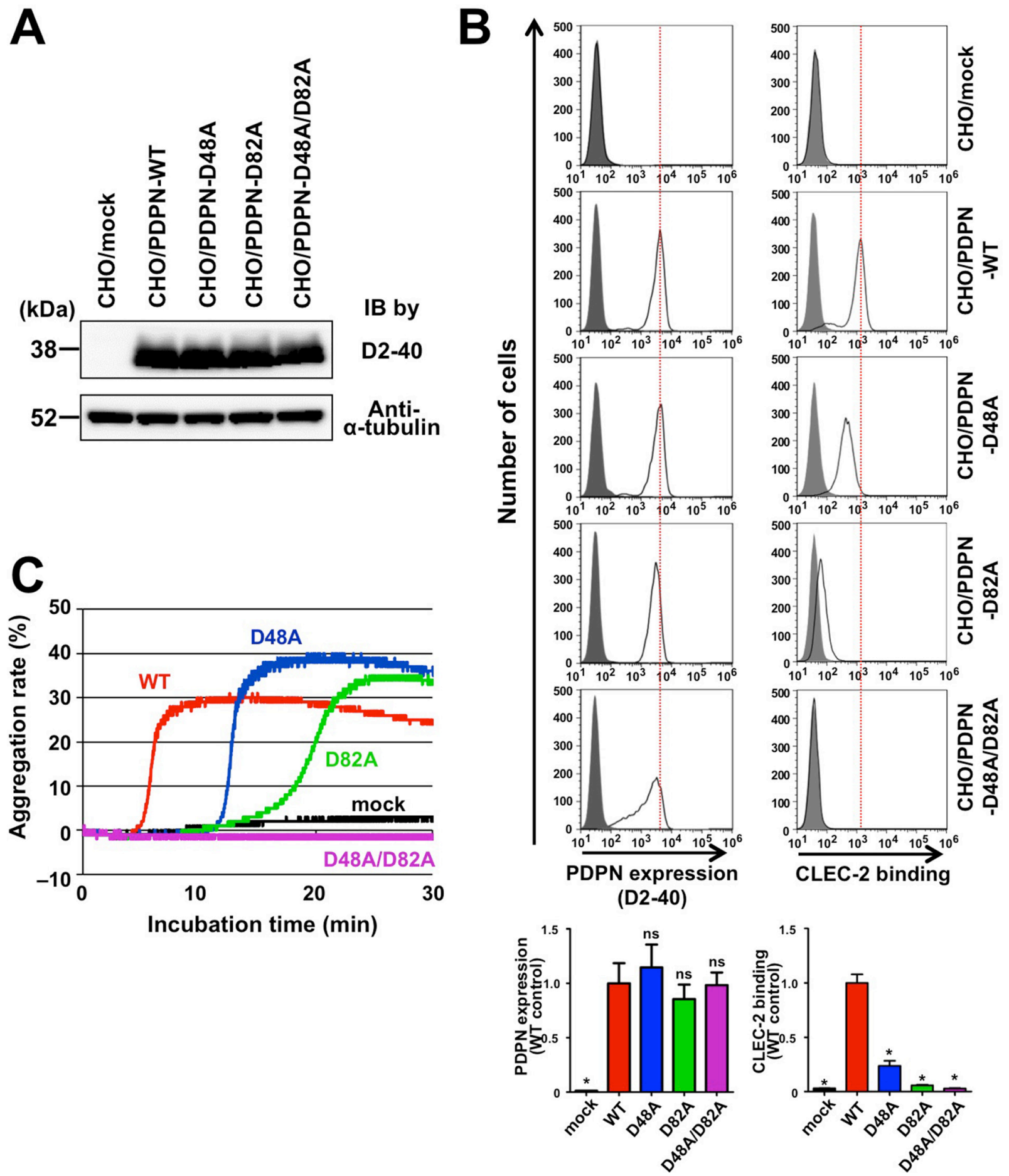

Figure 2: Involvement of PLAG4 domain in CLEC-2 binding and platelet aggregation. (A) CHO cells that had been stably transfected with empty vector (mock), WT-PDPN, or PLAG3/4 point mutants were lysed and immunoblotted with antibodies to podoplanin (D2-40) or $\alpha$-tubulin. (B) CHO cells that had been stably transfected with empty vector (mock), WT-PDPN, or PLAG3/4 point mutants were treated with control mouse IgG (closed areas) or anti-PDPN mAb (D2-40; open areas) for checking PDPN expression level (upper left panels), or with PBS (closed areas) or CLEC-2-(His) ${ }_{10}$ (open areas) for estimating CLEC-2-binding ability (upper right panels). After washing, cells were incubated with Alexa Flour 488-conjugated second antibody. The flow cytometry data (upper) and their quantitative graphs (lower) are shown. Each value in the lower graphs indicates mean $\pm \operatorname{SDs}(N=3)$ of the peak value normalized by that of PDPN-WT/CHO. $* P<0.05$ using Mann-Whitney $U$ test. ns, not significant. (C) $\mathrm{CHO}$ cells that had been stably transfected with empty vector (mock), WT-PDPN (WT), or PLAG3/4 point mutants (D48A, D82A, and D48A/D82A) were incubated with mouse PRP. The aggregation rate was estimated using an aggregometer. 
A

PDPN-AN24

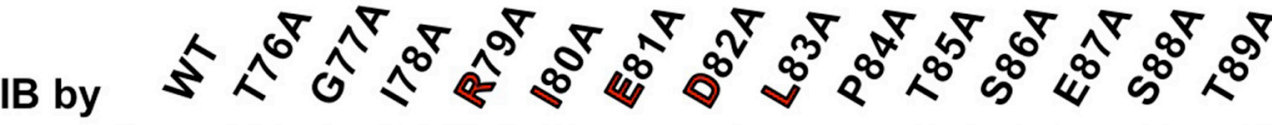

MS-1

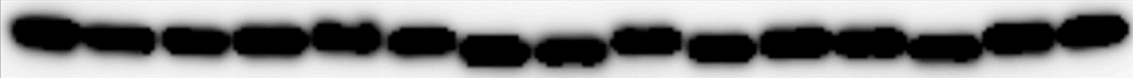

PG4D1

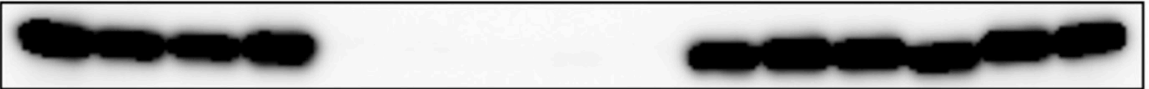

PG4D2

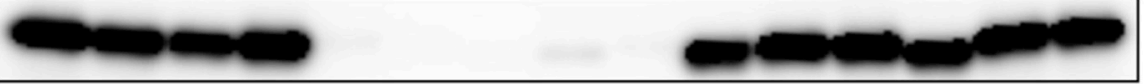

PG4D2

(long

exposure)

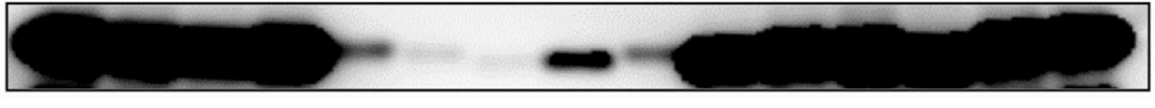

Anti-GST
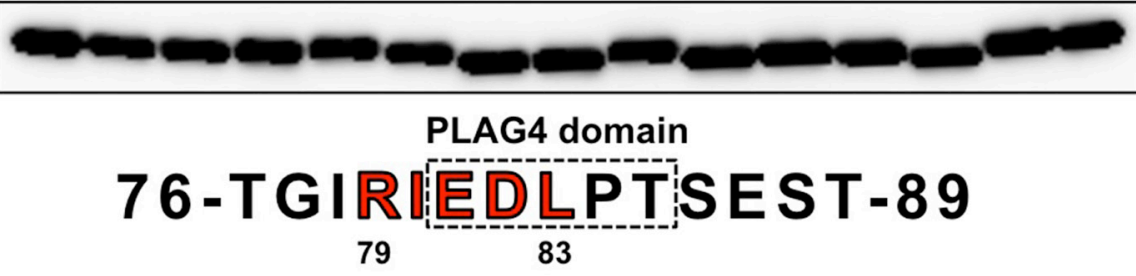

B
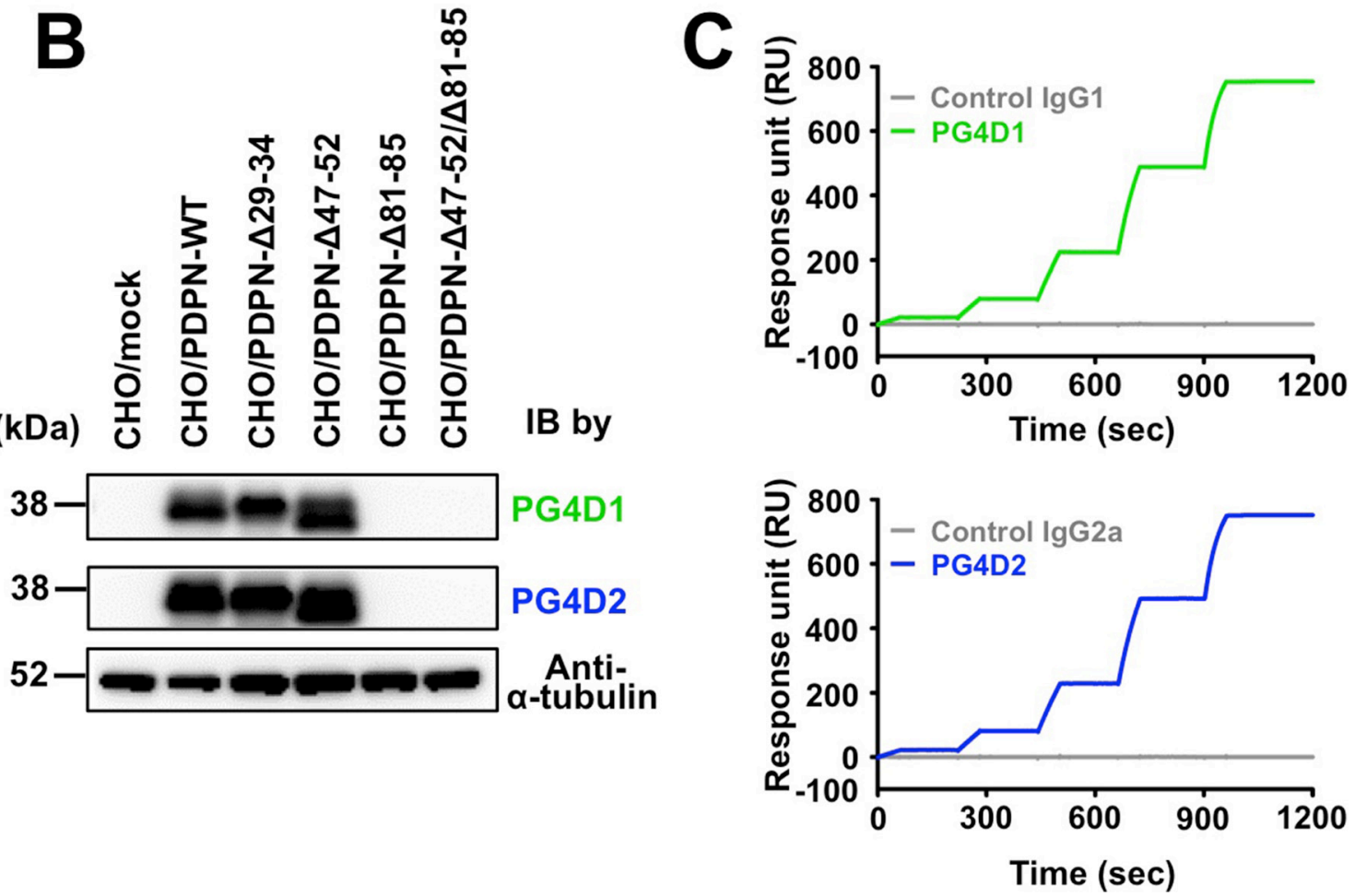

Figure 3: Establishment of PLAG4 domain-recognizing anti-podoplanin-neutralizing mAbs. (A) GST-tagged recombinant human $\Delta$ N24-podoplanin protein (WT) and its point mutants were expressed in E. coli. Cell lysates were electrophoresed and immunoblotted with antibodies to podoplanin (MS-1, PG4D1, and PG4D2) or GST. In the fourth panel, the image of PG4D2 immunoblotting after long exposure is shown. The predicted PG4D1 and PG4D2 mAb-recognition domain (79-RIEDL-83) is indicated by red bold letters. (B) CHO cells that had been stably transfected with empty vector (mock), PDPN-WT, or PLAG domain-deleted PDPN mutants were lysed and immunoblotted with antibodies to podoplanin (PG4D1 and PG4D2) or $\alpha$-tubulin. (C) Interaction between the human podoplanin protein immobilized on the CM-5 tip and control IgG1 (gray line, upper graph), the PG4D1 mAb (green line, upper graph), control IgG2a (gray line, lower graph), or the PG4D2 mAb (blue line, lower graph) was estimated by SPR analysis. Equilibrium dissociation constants $\left(K_{D}\right)$ of PG4D1 or PG4D2 mAbs on human podoplanin could not be calculated. 
concentration-dependent manner (Figure 4A). At higher concentrations, PG4D1 and PG4D2 mAbs exerted stronger inhibitory activities than our previously generated MS-1 mAb, which targets the PLAG2/PLAG3 domain [23]. Consistently, and similar to the MS- $1 \mathrm{mAb}$, masking the PLAG4 domain by PG4D1 and PG4D2 mAbs attenuated CHO/PDPN binding to recombinant CLEC-2 (Figure 4B).

To exclude the possibility that anti-PLAG4 mAbs showed the inhibition of podoplanin-CLEC-2 binding by modulating the PLAG3-mediated binding to CLEC-2, we performed a competition assay again using the D48A/ PLAG3 point mutant (Supplementary Figure S3B). PG4D1 and PG4D2 mAbs were able to neutralize CHO/PDPN-D48A binding to recombinant CLEC-2 in a dose-dependent manner and could almost completely suppress binding at the highest $\mathrm{mAb}$ concentration (100 $\mu \mathrm{g} / \mathrm{mL}$, Supplementary Figure S3B). To further confirm that PLAG3 and PLAG4 domains are independently associated with CLEC-2, we carried out competitive flow cytometric analyses using fluorescent dye (DyLight594)labeled PG4D2 and MS-1 mAbs (Figure 4C). The reaction of DyLight594-labeled MS-1 mAb to CHO/PDPN was attenuated by the preincubation of the cells with non-labeled MS-1 mAb but not by the non-labeled PG4D2 and vice versa (Figure $4 \mathrm{C}$ ). These results indicated that PG4D2 and MS-1 mAbs did not sterically hinder the mutual targeting PLAG domain and that masking one PLAG domain did not induce conformational changes affecting CLEC-2 interaction through the other PLAG domain. Therefore, PLAG3 and PLAG4 domains would independently bind to CLEC-2.

We previously reported that podoplanin-CLEC-2 interaction was essential for podoplanin-induced platelet aggregation and tumor metastasis $[3,6]$. We therefore examined the effect of anti-PLAG3 mAb MS-1 and anti-PLAG4 mAbs PG4D1 and PG4D2 on podoplanininduced platelet aggregation. The incubation of mouse platelets with CHO/PDPN cells induced platelet aggregation, and the aggregation starting time was delayed by adding MS-1, PG4D1, or PG4D2 mAb (Figure 5A). The platelet aggregation induced by D48A/PLAG3mutated $\mathrm{CHO} / \mathrm{PDPN}$ cells could be suppressed almost completely by adding anti-PLAG4 mAb PG4D2 but not by MS-1 mAb (Figure 5B). Similarly, the platelet aggregation induced by D82A/PLAG4-mutated CHO/ PDPN cells could be almost completely suppressed by adding anti-PLAG3 mAb MS-1 but not by PG4D2 mAb (Supplementary Figure S4). These results demonstrated that the PLAG4 domain possesses platelet aggregation ability in addition to PLAG3 domain in human podoplanin. We next performed an in vivo metastasis assay to verify the role of the PLAG4 domain. Podoplanininduced pulmonary metastasis was significantly blocked by a single administration of PG4D1 or PG4D2 mAb on the day before tumor inoculation, like MS-1 mAb (Figure 5C and 5D). These results indicate that the PLAG4 domain has a role in podoplanin-mediated metastasis formation, and blocking the interaction between PLAG4CLEC-2 would be a promising strategy for suppressing hematogenous metastasis.

\section{DISCUSSION}

We previously identified podoplanin/Aggrus/gp44 as a novel platelet aggregation-inducing factor and then investigated the domain critical to its platelet aggregationinducing activity [6]; we discovered that the EDXXVTPG motif is associated with its platelet-aggregating activity, therefore designated the domain as the PLAG domain [6]. Almost all mammalian podoplanins contain three tandem repeats of the PLAG domain at the extracellular domain (PLAG1-3) [12]. Consistent with our previous finding that $\mathrm{O}$-glycanase treatment attenuated the platelet-aggregating activity of podoplanin/gp44 [13], the Thr residue within the PLAG domain was found to be $O$-glycosylated [25], and the $O$-glycosylation is indispensable for exhibiting its platelet-aggregating activity. This was evidenced by the fact that substitution of $\mathrm{Thr}^{52}$ to Ala in the human PLAG3 domain (47-EDDVVT ${ }^{52}$ PG-54) attenuated the activity and that podoplanin expressed in glycosylationdeficient $\mathrm{CHO}$ mutants, Lec-2 or Lec-8, exhibited no platelet-aggregating activity $[6,14]$. Moreover, a synthetic podoplanin peptide containing human PLAG2 and PLAG3 domains exhibited platelet-aggregating activity only after $O$-glycosylation at the $\mathrm{Thr}^{52}$ residue [15]. A recent report on crystal structure revealed that $O$-glycans attached to $\mathrm{Thr}^{52}$ in addition to $\mathrm{Glu}^{47}$ and $\mathrm{Asp}^{48}$ residues in the human PLAG3 domain are associated with the binding to Arg residues in CLEC-2 [24]. Thus, the minimum structure essential for the binding to CLEC2 appeared to be two negatively charged amino acids following an $O$-glycosylated amino acid (i.e., EDXXXT). In the case of our newly identified PLAG4 domain (81-EDLPT ${ }^{85}$ SE-87), Thr $^{85}$ and Ser $^{86}$ residues appeared to be $O$-glycosylated since the NetOGlyc 4.0 Server [26] predicted a higher possibility of $O$-glycosylation at the $\mathrm{Thr}^{85}$ and $\mathrm{Ser}^{86}$ residues in the human PLAG4 domain than at the $\mathrm{Thr}^{52}$ residue in the human PLAG3 domain. Moreover, Zimmer et al. previously suggested glycosylation at the $\mathrm{Thr}^{92}$ residue within the canine PLAG4 domain (88-EDGPT ${ }^{92}$ QE-94) [25]. Therefore, $O$-glycosylated $\mathrm{Thr}^{85}$ and/or $\mathrm{Ser}^{86}$ in addition to negatively charged $\mathrm{Glu}^{81}$ and $\mathrm{Asp}^{82}$ residues in the human PLAG4 domain would be associated with the binding to CLEC-2 and the platelet-aggregating activity of podoplanin. Based on the low homology of the $\mathrm{Ser}^{86}$ residue among mammalian podoplanin, $O$-glycans attached to the $\mathrm{Ser}^{86}$ residue in the human PLAG4 domain may not be required for CLEC-2 binding but may contribute to robust PLAG4-CLEC-2 interaction. At least, we indicated the contribution of $\mathrm{Thr}^{85}$ residue to CLEC-2 binding using its Ala-substituted mutant (Supplementary Figure S2B). The 

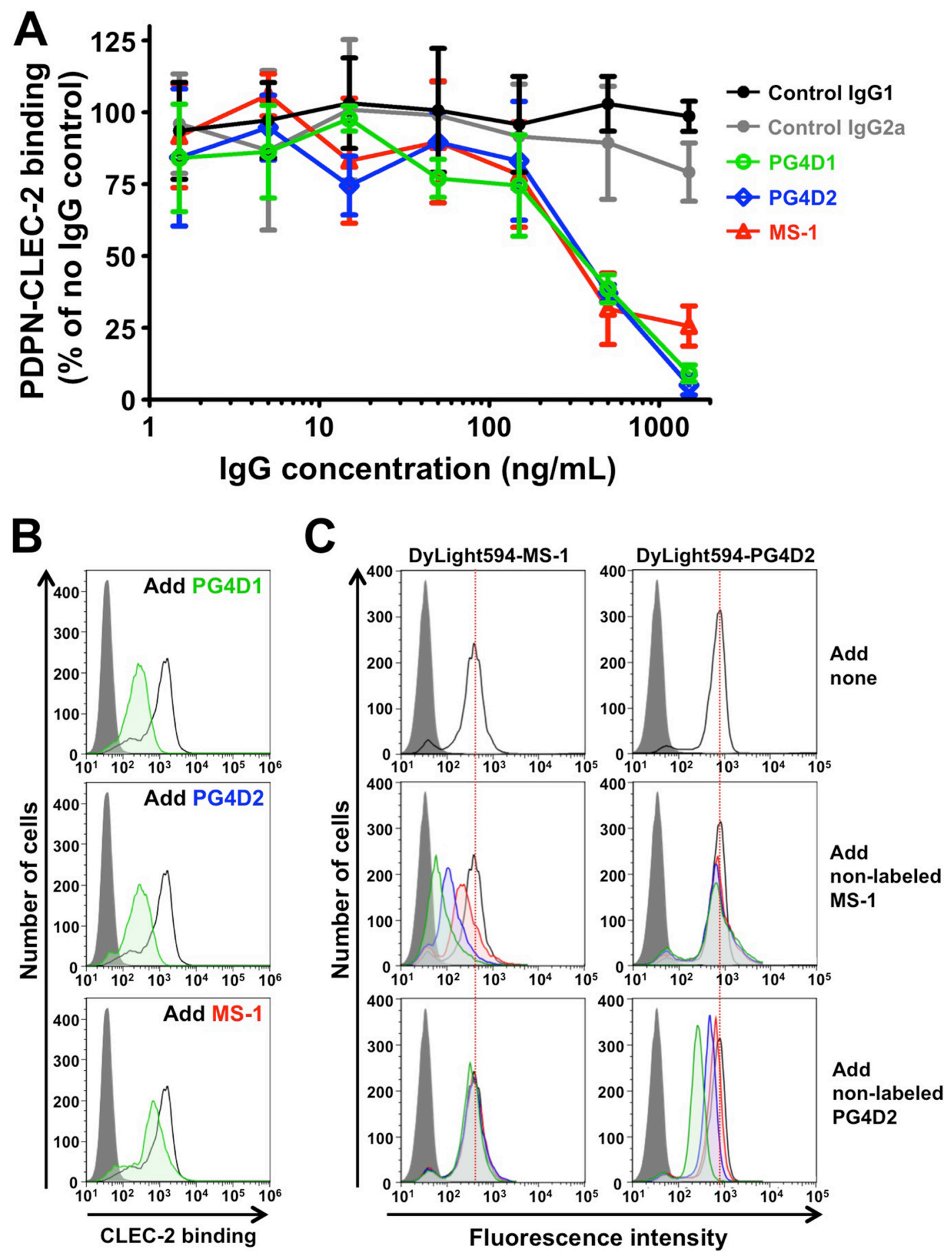

Figure 4: Neutralization of podoplanin-CLEC-2 binding by anti-PLAG4 mAbs PG4D1 and PG4D2. (A) PodoplaninFc protein (1 ng/well) was incubated with the indicated concentrations of control or anti-podoplanin antibodies in a 96-well plate. Next, CLEC-2-(His) $)_{10}$ protein (30 ng/well) was added to each well. After incubation with alpha screen beads, podoplanin-CLEC-2 binding intensity was measured as a fluorescence signal. No IgG control was taken as $100 \%$. The graph shows mean \pm SDs $(N=3)$. (B) $\mathrm{CHO} / \mathrm{PDPN}-\mathrm{WT}$ cells were incubated with $100 \mu \mathrm{g} / \mathrm{mL}$ of control IgG1/IgG2a or anti-podoplanin (PG4D1, PG4D2, or MS-1) antibodies, followed by incubation with $0.4 \mu \mathrm{g} / \mathrm{mL}$ of CLEC-2-(His) ${ }_{10}$ protein (open areas: control IgG-treated samples; green areas: PG4D1-, PG4D2-, or MS-1-treated samples). After washing, cells were further incubated with Alexa Flour 488-conjugated anti-penta-His second antibody. CLEC-2 binding was measured by flow cytometry. Grey areas indicate the fluorescence intensity of CLEC-2-non-treated samples. (C) CHO/PDPN-WT cells were incubated with $10 \mu \mathrm{g} / \mathrm{mL}$ of DyLight594-conjugated MS-1 or PG4D2 mAb (top panels). In some experiments, cold MS-1 or PG4D2 mAb (green areas: $10 \mu \mathrm{g} / \mathrm{mL}$; blue areas: $3 \mu \mathrm{g} / \mathrm{mL}$; red areas: $1 \mu \mathrm{g} / \mathrm{mL}$ ) were co-treated with $10 \mu \mathrm{g} / \mathrm{mL}$ of DyLight594-conjugated antibodies (middle and bottom panels). After washing, fluorescence intensity was measured by flow cytometry. 
number of amino acids intervening between negatively charged Glu-Asp residues and $O$-glycosylated Thr residue is two in the PLAG4 domain and three in the PLAG1-3 domains (Figure 1E). Moreover, the EDXXT motif in the PLAG4 domain is highly conserved among mammals (36/42 species from multiple sequence alignment analysis; Supplementary Figure S1), similar to the EDXXXT motif in PLAG1 and PLAG3 (40/42 and 38/42 species, respectively). Therefore, we find it appropriate to revise the PLAG consensus sequence to $\mathrm{ED}(\mathrm{X}) \mathrm{XXT}$, as shown in Figure 1E.

The substitution of positively charged Arg residues in CLEC-2 has been reported to suppress the binding to podoplanin almost completely [24]. Although the

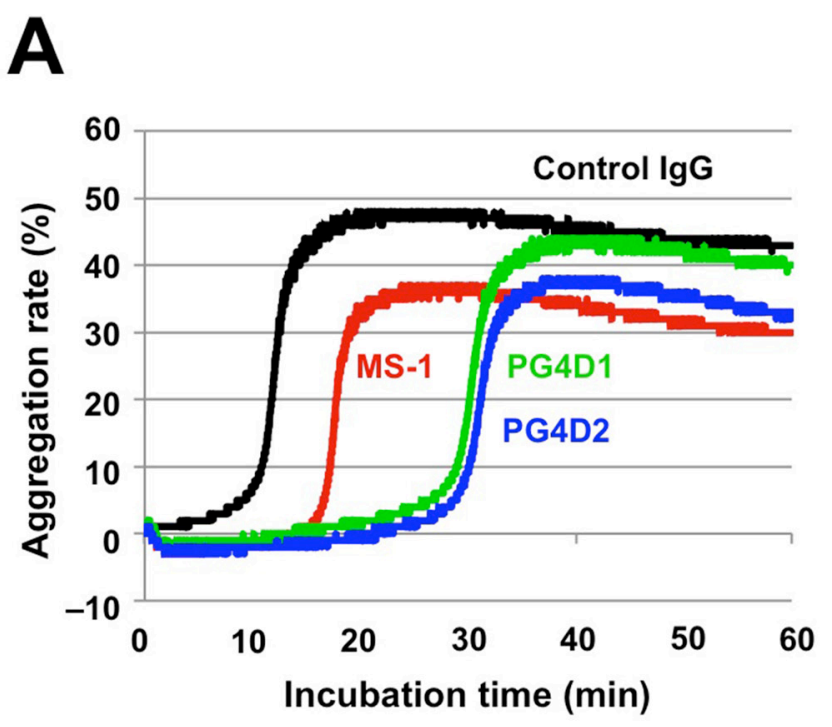

B

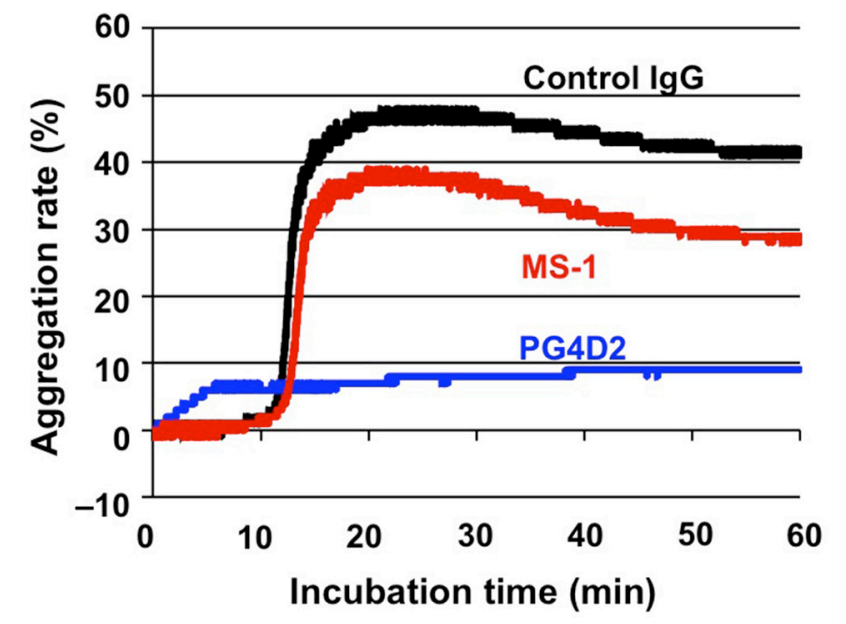

C
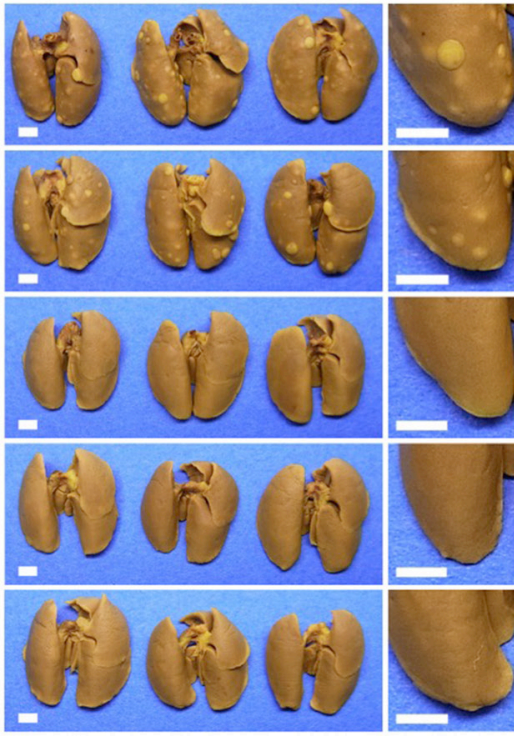

Treated by D

Control IgG1

Control IgG2a

PG4D1

PG4D2

MS-1

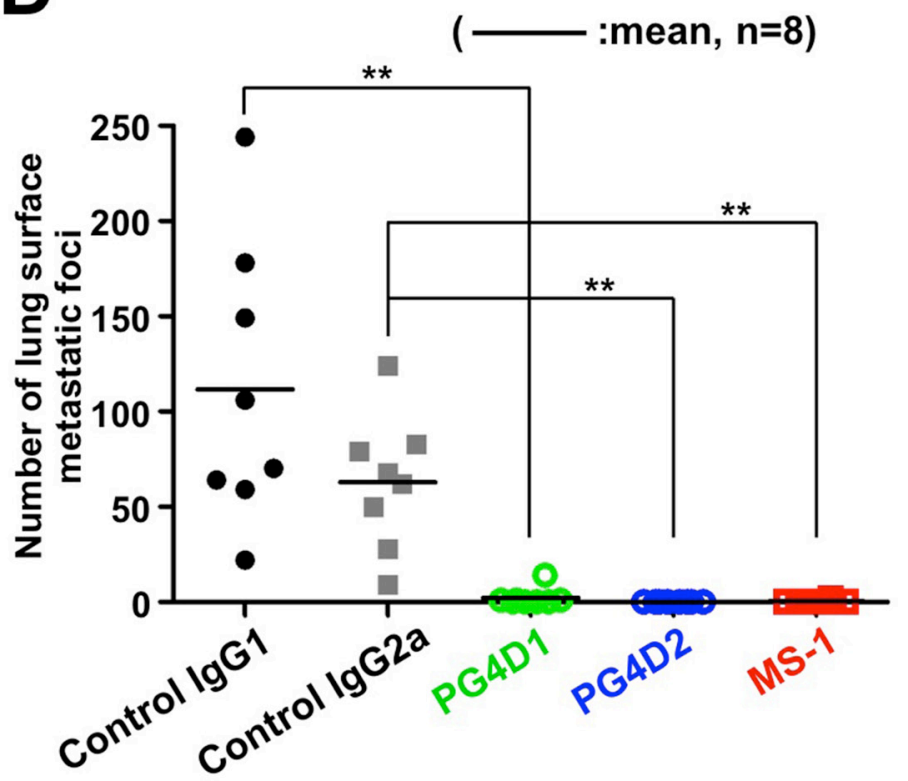

Figure 5: Inhibition of podoplanin-mediated platelet aggregation and pulmonary metastasis by anti-PLAG4 mAbs PG4D1 and PG4D2. (A and B) CHO/PDPN-WT cells (A) or CHO/PDPN-D48A cells (B) were incubated with $10 \mu \mathrm{g} / \mathrm{mL}$ of the indicated antibodies followed by incubation with mouse PRP. The aggregation rate was estimated using an aggregometer. (C and D) $\mathrm{BALB} / \mathrm{c}-n u / n u$ mice were intravenously injected with $10 \mu \mathrm{g} /$ mouse of the indicated antibodies. The next day, CHO/PDPN-WT cells $\left(2.5 \times 10^{5}\right.$ cells/mouse $)$ were intravenously inoculated into the mice. After 19 days of tumor inoculation, the mice were euthanized, and lung surface metastatic foci were counted. The representative pictures of lungs are shown (C). Areas surrounded by boxes are magnified (right pictures). Bars, $2 \mathrm{~mm}$. The number of metastatic foci is shown (D). Bars, mean $(N=8) . * * P<0.01$ using the Mann-Whitney $U$ test. 
deletion of the PLAG3 or PLAG4 domain weakened this interaction, the deletion of both PLAG3 and PLAG4 domains resulted in a complete loss of podoplanin-CLEC-2 interaction (Figure 1C). Furthermore we have confirmed $\mathrm{Glu}^{81}, \mathrm{Asp}^{82}$, and $\mathrm{Thr}^{85}$ residues in the PLAG4 domain, with the equivalent positions of $\mathrm{Glu}^{47}, \mathrm{Asp}^{48}$, and $\mathrm{Thr}^{52}$ residues in the PLAG3 domain, as essential for CLEC2 binding (Figure 2B and Supplementary Figure S2B). These results suggest that the human PLAG4 and PLAG3 domains share a binding site on CLEC-2. Because podoplanin could coincidentally form a complex with an antibody (150 kDa) larger than CLEC-2 (35 kDa), (Figure 4C), it is possible that one molecule of podoplanin could bind to two molecules of CLEC-2 at the same time. This possibility is an advantage for Src and Syk signal activation via a CLEC-2 dimer [27]. Two CLEC-2-binding sites located on the podoplanin molecule may contribute to CLEC-2 clustering and to fast signal transduction [28]. Although the monomer podoplanin-Fc protein alone could not induce platelet aggregation, the addition of a secondary antibody that enhanced CLEC- 2 crosslinking and oligomerization could trigger platelet aggregation (data not shown). These results suggest that the condensation of CLEC-2 in the cell-platelet adhesion area would be essential for transmitting platelet-aggregating signals via CLEC-2.

The actual role sharing between the PLAG3 and PLAG4 domains in CLEC-2 binding remains unknown. PLAG4 deletion or PLAG4 mutation resulted in a drastic decrease in podoplanin-CLEC-2 binding when compared with PLAG3 deletion or PLAG3 mutation (Figure $1 \mathrm{C}$ and 2B). Moreover, the anti-PLAG4 mAbs (PG4D1 and PG4D2) exerted stronger inhibitory activity against podoplanin-CLEC-2 binding than did the anti-PLAG3 mAb (MS-1) did (Figure 4A). These results suggest the importance of the PLAG4 domain in podoplanin-CLEC-2 binding. However, we could not exclude the possibility that each cell uses the PLAG3 and PLAG4 domains according to cell context or microenvironment. In fact, the anti-PLAG3 $\mathrm{mAb}$ preferentially recognized podoplanin expressed on H226, PC-10, and KYSE70 cells, whereas anti-PLAG4 $\mathrm{mAbs}$ preferentially recognized podoplanin expressed on UM-UC-5 and HT1080 cells (Supplementary Figure S5). As no mutation was found in podoplanin expressed in these cell lines, post-translational modifications, i.e., glycosylation, and interaction with other molecules, etc. may affect the reactivity of the mAbs. These notions were supported by a recent report that the anti-PLAG4 mAb preferentially recognized metastasized osteosarcomas [29].

Our established anti-PLAG4 neutralizing mAbs, PG4D1 and PG4D2, exhibited a high affinity to human podoplanin with very low $K_{\mathrm{D}}$ values (less than $0.3 \mathrm{nM}$ ). The single administration of the anti-PLAG4 mAbs into mice could almost completely suppress hematogenous metastasis of CHO/PDPN cells. Since a PG4D1 mAb of murine IgG1 subclass exhibiting low ADCC/CDC activity and a PG4D2 mAb of murine IgG2a subclass having strong $\mathrm{ADCC} / \mathrm{CDC}$ activity could suppress pulmonary metastasis, ADCC/CDC activity would not be associated with the metastasis-inhibitory function of the mAbs, similarly to the anti-PLAG3 mAb MS-1 [23]. Considering the complete metastasis suppression by only a single administration of anti-PLAG3 mAb MS-1 (Figure 5C and 5D) [23], the transient suppression of podoplanin-CLEC-2 binding would be sufficient to suppress podoplanin-mediated hematogenous metastasis. With regard to tumor suppression, $\mathrm{ADCC} / \mathrm{CDC}$ activity may be partly involved in the anti-tumor activity of the anti-podoplanin antibodies because an anti-PLAG3 mAb MS-1 of murine IgG2a subclass having strong ADCC/ CDC activity, but not an anti-PLAG3 mAb P2-0 of murine IgG1 subclass exhibiting low ADCC/CDC activity, could attenuate $\mathrm{CHO} / \mathrm{PDPN}$ proliferation in nude mice [23]. Of course, the inhibition of podoplanin-CLEC-2 binding would be sufficient to suppress the proliferation of some tumors in vivo [23]. However, the interruption of $\mathrm{mAb}$ administration resulted in recurrence of the tumors [23]. These results indicate that either the use of $\mathrm{mAb}$ possessing strong $\mathrm{ADCC} / \mathrm{CDC}$ activity or the complete inhibition of podoplanin-CLEC-2 binding by the combined administration of anti-PLAG3 and antiPLAG4 mAbs may be required for inducing complete tumor remission. As podoplanin is known to be expressed in normal tissue, including lymphatic vessels, kidney podocytes, mesothelium, and alveolar epithelium, the complete blockade of podoplanin-CLEC-2 binding by the co-administration of anti-PLAG3 and anti-PLAG4 mAbs possessing no ADCC/CDC activity would be preferable as anti-tumor agents with minimal side effects.

In this report, we succeeded in identifying the novel PLAG4 domain that is associated with the binding to CLEC-2 and platelet aggregation. Based on the PLAG4 domain sequence, we would propose $\mathrm{ED}(\mathrm{X}) \mathrm{XXT}$ as the revised PLAG domain consensus sequence. Our finding is expected to shed light on the clinical development of podoplanin-targeting cancer therapy.

\section{MATERIALS AND METHODS}

\section{Cells and cell culture conditions}

CHO cells were purchased from the American Type Culture Collection (ATCC, Manassas, VA, USA) and cultured in PRMI 1640 medium (Wako, Osaka, Japan) containing 10\% FBS (Sigma-Aldrich, St Louis, MO, USA). Stable podoplanin-expressing clones were established as described previously [6] and cultured in a medium containing $250 \mu \mathrm{g} / \mathrm{mL}$ of G418 (Life Technologies, Carlsbad, CA, USA). 


\section{Plasmid construction}

The pcDNA3 vector containing WT human podoplanin cDNA (pcDNA3-podoplanin) was established as described previously [6]. pcDNA3 vectors containing podoplanin deletion mutated cDNAs (PDPN- $\Delta 29-34$, PDPN- $\Delta 47-52$, PDPN- $\Delta 81-85$, and PDPN- $\Delta 47-52 / \Delta 81-85$ ) or podoplanin point-mutated cDNAs (PDPN-G45A, PDPN-D48A, PDPND48N, PDPN-D48E, PDPN-D49A, PDPN-D82A, and PDPND48A/D82A) were generated from pcDNA3-podoplanin as a template using the QuikChange site-directed mutagenesis kit (Agilent Technology, Santa Clara, CA, USA). pGEX-6P-3 vectors (GE Healthcare, Buckinghamshire, UK) containing signal peptide-deleted podoplanin cDNA (PDPN- $\Delta \mathrm{N} 24$ ) or its point-mutated cDNAs were constructed as described previously [22].

\section{Animals}

Female BALB/c, BALB/c-nu/nu, and Jcl:ICR mice were purchased from Charles River (Kanagawa, Japan). All animal procedures were performed according to the protocols approved by the Japanese Foundation for Cancer Research Animal Care and Use Committee.

\section{Multiple sequence alignment and window analysis}

All podoplanin protein sequences were obtained from NCBI's reference sequence database (http:// www.ncbi.nlm.nih.gov/refseq/). For the alignment of podoplanin sequences, sequences including multiple gaps were excluded, and typical experimental animal species and representative species of each family were selected. The selected 42 sequences are presented as supplementary information (Supplementary Figure S1). Sequences were aligned using the muscle alignment algorithm available in the MEGA6 software [30]. Sliding-window analysis was performed using the AL2CO program [31]. Hydropathy plots were generated using Kyte and Doolittle amino acid hydropathy scores [32].

\section{Immunoblotting}

Cells were lysed in TENSV buffer $(50 \mathrm{mM}$ Tris- $\mathrm{HCl}$ [pH 7.5], $100 \mathrm{mM} \mathrm{NaCl}, 2 \mathrm{mM}$ EDTA, 1\% NP-40, 0.1\% aprotinin, and $2 \mathrm{mM}$ phenylmethylsulfonyl fluoride) and electrophoresed followed by the transfer to a PDVF membrane (Millipore, Billerica, Massachusetts, USA). The GST-tagged human PDPN- $\triangle \mathrm{N} 24$ and its point mutants were produced in BL21 (DE3) E. coli (Thermo Fisher Scientific, Waltham, MA, USA), and the cell pellets were boiled in SDS sample buffer for immunoblots. The membranes were incubated with antibodies to podoplanin (D2-40, AbD Serotec, Oxford, UK; FL-162, Santa Cruz Biotechnology, Santa Cruz, CA, USA; MS-1; P2-0; PG4D1; and PG4D2), $\alpha$-tubulin (Sigma-Aldrich, St Louis, MO, USA), and GST (Abcam, Cambridge, UK).

\section{Flow cytometry}

Cells were harvested and treated with $1 \mu \mathrm{g} / \mathrm{ml}$ of antipodoplanin antibodies or control mouse IgG (Sigma-Aldrich, St Louis, MO, USA), following incubation with $4 \mu \mathrm{g} / \mathrm{mL}$ of Alexa Flour 488-conjugated anti-mouse $\operatorname{IgG}(\mathrm{H}+\mathrm{L})$ (Thermo Fisher Scientific, Waltham, MA, USA). For CLEC-2-binding analysis, cells were incubated with $0.4 \mu \mathrm{g} / \mathrm{mL}$ of (His) ${ }_{10}$-tagged human CLEC-2 protein (R \& D Systems, Minneapolis, MN, USA), following incubation with 1/500 dilutions of Alexa Flour 488-conjugated anti-penta-His antibody (QIAGEN, Venlo, Netherlands). Flow cytometric analyses were performed using Cytomics FC500 flow cytometry system (Beckman Coulter, CA, USA). In some experiments, MS-1 and PG4D2 mAbs were used as primary antibodies, following the conjugation of DyLight594 using the DyLight antibody labeling kit (Thermo Fisher Scientific, Waltham, MA, USA).

\section{Platelet aggregation assay}

Murine whole blood was drawn by cardiac puncture from Jcl:ICR mice terminally anesthetized with sevoflurane and taken with heparin solution. Platelet-rich plasma (PRP) was obtained from the supernatant of whole blood by centrifugation at $120 \mathrm{xg}$ for $8 \mathrm{~min}$ twice. Platelets were collected from the pellets of PRP by centrifugation at $500 \mathrm{xg}$ for $10 \mathrm{~min}$, and the supernatant was stocked as platelet-poor plasma (PPP). Washed platelets were prepared by washing the pellets with the modified Tyrode's buffer $(137 \mathrm{mM} \mathrm{NaCl}, 11.9 \mathrm{mM} \mathrm{NaHCO}, 0.4 \mathrm{mM}$ $\mathrm{Na}_{2} \mathrm{HPO}_{4}, 2.7 \mathrm{mM} \mathrm{KCl}, 1.1 \mathrm{mM} \mathrm{MgCl}$, and $5.6 \mathrm{mM}$ glucose, $\mathrm{pH}$ 7.3) followed by centrifugation under the same conditions and resuspended in the modified Tyrode's buffer at a concentration of $1 \times 10^{8} / \mathrm{mL}$. Prior to the assay, $10 \%$ PPP and $400 \mu \mathrm{M} \mathrm{CaCl}_{2}$ were supplied to the washed platelets. Next, the washed platelets were incubated with cell suspension $\left(1 \times 10^{8}\right.$ cells $\left./ \mathrm{mL}\right)$. For the antibody inhibition assay, cells were incubated with $10 \mu \mathrm{g} / \mathrm{mL}$ of $\mathrm{mAb}$ or control IgG for $30 \mathrm{~min}$ on ice before incubation with platelets. The platelet aggregation rate was examined using a platelet aggregometer (MCM HEMA TRACER 313M; SSR Engineering, Kanagawa, Japan).

\section{Hybridoma production and antibody purification}

A human podoplanin cDNA region encoding amino acids 76-89 (226-267 bp) was cloned and tandemly connected 12 and 40 times. These cDNA fragments were inserted into a pGEX-6P-3 vector (GE Healthcare, Buckinghamshire, UK). Next, the GST-tagged human podoplanin peptide (76-89 aa) produced in BL21 (DE3) E. coli was purified using glutathione sepharose. Six-week-old female BALB/c mice were injected with the GST-tagged peptide as an immunogen in conjugation with Titer MAX Gold adjuvant (Titer MAX, Norcross, GA, USA). Further, intraperitoneal immunization was performed intermittently for two months. Mice were 
euthanized, and splenocytes were fused with mouse myeloma P3U1 cells using PEG4000 (Merck, Whitehouse Station, NJ, USA). Hybridoma screening and antibody purification from ascites were performed as described previously [22]. IgG isotypes were identified using the Mouse Monoclonal Antibody Isotyping Test Kit (AbD Serotec, Oxford, UK).

\section{SPR analysis}

SPR analysis was performed using Biacore X100 (GE healthcare, Buckinghamshire, UK). Recombinant human podoplanin-Fc protein ( $\mathrm{R} \& \mathrm{D}$ Systems, Minneapolis, MN, USA) was immobilized on a CM5 sensor chip (GE Healthcare, Buckinghamshire, UK). Final levels of immobilization were approximately 562.2 or 562.6 response units (measure conditions of PG4D1 or PG4D2, respectively). Five concentrations of PG4D1, PG4D2 mAbs, and their control mouse IgGs were flowed over the chip in the single cycle kinetics. Sensorgrams were fit by global analysis using the Biacore X100 evaluation software. Efforts toward determining the equilibrium dissociation constant $\left(K_{\mathrm{D}}\right)$ were decided using the bivalent binding analyte model.

\section{Alpha screen}

Podoplanin-Fc protein (1 ng/well) was firstly incubated with PG4D1, PG4D2, and MS-1 mAbs, or control IgG1 and IgG2a in a 96-well plate. Next, (His) ${ }_{10}$-tagged human CLEC-2 protein (30 ng/well) was added to the mixture, following the addition of alpha screen beads (Nickel Chelate Donor beads and Protein A Acceptor beads; Perkin Elmer, Waltham, MA, USA). After incubation for $30 \mathrm{~min}$ at RT, binding intensity was measured as a fluorescence signal using the EnVision plate reader (Perkin Elmer, Waltham, MA, USA).

\section{Experimental pulmonary metastasis}

Five-week-old female BALB/c-nu/nu mice were intravenously inoculated with antibodies (10 $\mu \mathrm{g} / \mathrm{mouse})$ the day before cell injection. CHO/PDPN cells were resuspended in Hanks' Balanced Salt Solution (HBSS, Gibco, Thermo Fisher Scientific, Waltham, MA, USA) and intravenously injected into mice $\left(2.5 \times 10^{5}\right.$ cells/mouse $)$. Eighteen days following $\mathrm{CHO} / \mathrm{PDPN}$ injection, the mice were euthanized, and their lungs were stained with the saturated picric acid solution. Surface lung metastatic foci were counted.

\section{Statistical analysis}

A Mann-Whitney $U$ test was performed to determine the statistical significance in flow cytometry and metastasis assays. Significant probability $(P)$ values are shown as $* P<0.05$, and $* * P<0.01$. All statistical tests were two-sided.

\section{ACKNOWLEDGMENTS AND FUNDINGS}

This study was performed as a research program of the Project for Development of Innovative Research on Cancer Therapeutics (P-DIRECT, No. 14056017), Japan Agency for Medical Research and development (AMED), and was supported in part by a Grant-in-Aid for Challenging Exploratory Research from JSPS, Japan (Grant No. 26640108; to N.F.), by SRF (to N.F.), and by the Princess Takamatsu Cancer Research Fund (Grant No. 13-24519; to N.F.).

\section{CONFLICTS OF INTEREST}

The authors have no conflicts of interest.

\section{REFERENCES}

1. Gay LJ, Felding-Habermann B. Contribution of platelets to tumour metastasis. Nat Rev Cancer. 2011; 11:123-134.

2. Gasic GJ, Gasic TB, Stewart CC. Antimetastatic effects associated with platelet reduction. Proc Natl Acad Sci USA. $1968 ; 61: 46-52$.

3. Kunita A, Kashima TG, Morishita Y, Fukayama M, Kato Y, Tsuruo T, Fujita N. The platelet aggregation-inducing factor aggrus/podoplanin promotes pulmonary metastasis. Am J Pathol. 2007; 170:1337-1347.

4. Klerk CP, Smorenburg SM, Otten HM, Lensing AW, Prins MH, Piovella F, Prandoni P, Bos MM, Richel DJ, van Tienhoven G, Buller HR. The effect of low molecular weight heparin on survival in patients with advanced malignancy. J Clin Oncol. 2005; 23:2130-2135.

5. Lazo-Langner A, Goss GD, Spaans JN, Rodger MA. The effect of low-molecular-weight heparin on cancer survival. A systematic review and meta-analysis of randomized trials. J Thromb Haemost. 2007; 5:729-737.

6. Kato Y, Fujita N, Kunita A, Sato S, Kaneko M, Osawa M, Tsuruo T. Molecular identification of Aggrus/T1alpha as a platelet aggregation-inducing factor expressed in colorectal tumors. J Biol Chem. 2003; 278:51599-51605.

7. Martin-Villar E, Scholl FG, Gamallo C, Yurrita MM, Munoz-Guerra M, Cruces J, Quintanilla M. Characterization of human PA2.26 antigen (T1alpha-2, podoplanin), a small membrane mucin induced in oral squamous cell carcinomas. Int J Cancer. 2005; 113:899-910.

8. Kimura N, Kimura I. Podoplanin as a marker for mesothelioma. Pathol Int. 2005; 55:83-86.

9. Mishima K, Kato Y, Kaneko MK, Nishikawa R, Hirose T, Matsutani M. Increased expression of podoplanin in malignant astrocytic tumors as a novel molecular marker of malignant progression. Acta Neuropathol. 2006; 111: 483-488.

10. Kunita A, Kashima TG, Ohazama A, Grigoriadis AE, Fukayama M. Podoplanin is regulated by AP-1 and 
promotes platelet aggregation and cell migration in osteosarcoma. Am J Pathol. 2011; 179:1041-1049.

11. Takagi S, Oh-hara T, Sato S, Gong B, Takami M, Fujita N. Expression of Aggrus/podoplanin in bladder cancer and its role in pulmonary metastasis. Int J Cancer. 2014; 134: 2605-2614.

12. Kaneko MK, Kato Y, Kitano T, Osawa M. Conservation of a platelet activating domain of Aggrus/podoplanin as a platelet aggregation-inducing factor. Gene. 2006; 378: 52-57.

13. Toyoshima $M$, Nakajima $M$, Yamori $T$, Tsuruo $T$. Purification and characterization of the platelet-aggregating sialoglycoprotein gp44 expressed by highly metastatic variant cells of mouse colon adenocarcinoma 26. Cancer Res. 1995; 55:767-773.

14. Kaneko M, Kato Y, Kunita A, Fujita N, Tsuruo T, Osawa M. Functional sialylated O-glycan to platelet aggregation on Aggrus (T1alpha/Podoplanin) molecules expressed in Chinese hamster ovary cells. J Biol Chem. 2004; 279:38838-38843.

15. Amano K, Chiba Y, Kasahara Y, Kato Y, Kaneko MK, Kuno A, Ito H, Kobayashi K, Hirabayashi J, Jigami Y, Narimatsu H. Engineering of mucin-type human glycoproteins in yeast cells. Proc Natl Acad Sci U S A. 2008; 105:3232-3237.

16. Kaneko MK, Kato Y, Kameyama A, Ito H, Kuno A, Hirabayashi J, Kubota T, Amano K, Chiba Y, Hasegawa Y, Sasagawa I, Mishima K, Narimatsu H. Functional glycosylation of human podoplanin: glycan structure of platelet aggregation-inducing factor. FEBS Lett. 2007; 581:331-336.

17. Suzuki-Inoue K, Fuller GL, Garcia A, Eble JA, Pohlmann S, Inoue O, Gartner TK, Hughan SC, Pearce AC, Laing GD, Theakston RD, Schweighoffer E, Zitzmann N, et al. A novel Syk-dependent mechanism of platelet activation by the C-type lectin receptor CLEC-2. Blood. 2006; 107:542-549.

18. Suzuki-Inoue K, Kato Y, Inoue O, Kaneko MK, Mishima K, Yatomi Y, Yamazaki Y, Narimatsu H, Ozaki Y. Involvement of the snake toxin receptor CLEC-2, in podoplaninmediated platelet activation, by cancer cells. J Biol Chem. 2007; 282:25993-26001.

19. Suzuki-Inoue K. Essential in vivo roles of the platelet activation receptor CLEC-2 in tumour metastasis, lymphangiogenesis and thrombus formation. J Biochem. 2011; 150:127-132.

20. Suzuki-Inoue K, Inoue O, Ding G, Nishimura S, Hokamura K, Eto K, Kashiwagi H, Tomiyama Y, Yatomi Y, Umemura K, Shin Y, Hirashima M, Ozaki Y. Essential in vivo roles of the C-type lectin receptor CLEC-2: embryonic/neonatal lethality of CLEC-2-deficient mice by blood/lymphatic misconnections and impaired thrombus formation of CLEC2-deficient platelets. J Biol Chem. 2010; 285:24494-24507.

21. Kato Y, Kaneko MK, Kuno A, Uchiyama N, Amano K, Chiba Y, Hasegawa Y, Hirabayashi J, Narimatsu H, Mishima K, Osawa M. Inhibition of tumor cell-induced platelet aggregation using a novel anti-podoplanin antibody reacting with its platelet-aggregation-stimulating domain. Biochem Biophys Res Commun. 2006; 349:1301-1307.

22. Nakazawa Y, Takagi S, Sato S, Oh-hara T, Koike S, Takami M, Arai H, Fujita N. Prevention of hematogenous metastasis by neutralizing mice and its chimeric anti-Aggrus/podoplanin antibodies. Cancer Sci. 2011; 102:2051-2057.

23. Takagi S, Sato S, Oh-hara T, Takami M, Koike S, Mishima Y, Hatake K, Fujita N. Platelets promote tumor growth and metastasis via direct interaction between Aggrus/podoplanin and CLEC-2. PLoS One. 2013; 8:e73609.

24. Nagae M, Morita-Matsumoto K, Kato M, Kaneko MK, Kato Y, Yamaguchi Y. A platform of C-type lectin-like receptor CLEC-2 for binding O-glycosylated podoplanin and nonglycosylated rhodocytin. Structure. 2014; 22: 1711-1721.

25. Zimmer G, Lottspeich F, Maisner A, Klenk HD, Herrler G. Molecular characterization of gp40, a mucin-type glycoprotein from the apical plasma membrane of MadinDarby canine kidney cells (type I). Biochem J. 1997; 326:99-108.

26. Steentoft C, Vakhrushev SY, Joshi HJ, Kong Y, VesterChristensen MB, Schjoldager KT, Lavrsen K, Dabelsteen S, Pedersen NB, Marcos-Silva L, Gupta R, Bennett EP, Mandel U, et al. Precision mapping of the human O-GalNAc glycoproteome through SimpleCell technology. EMBO J. 2013; 32:1478-1488.

27. Watson AA, Christou CM, James JR, Fenton-May AE, Moncayo GE, Mistry AR, Davis SJ, Gilbert RJ, Chakera A, O'Callaghan CA. The platelet receptor CLEC-2 is active as a dimer. Biochemistry. 2009; 48:10988-10996.

28. Pollitt AY, Poulter NS, Gitz E, Navarro-Nunez L, Wang YJ, Hughes CE, Thomas SG, Nieswandt B, Douglas MR, Owen DM, Jackson DG, Dustin ML, Watson SP. Syk and Src family kinases regulate C-type lectin receptor 2 (CLEC2)-mediated clustering of podoplanin and platelet adhesion to lymphatic endothelial cells. J Biol Chem. 2014; 289: 35695-35710.

29. Kaneko MK, Oki H, Ogasawara S, Takagi M, Kato Y. Anti-podoplanin Monoclonal Antibody LpMab-7 Detects Metastatic Lesions of Osteosarcoma. Monoclon Antib Immunodiagn Immunother. 2015; 34:154-161.

30. Tamura K, Stecher G, Peterson D, Filipski A, Kumar S. MEGA6: Molecular Evolutionary Genetics Analysis version 6.0. Mol Biol Evol. 2013; 30:2725-2729.

31. Pei J, Grishin NV. AL2CO: calculation of positional conservation in a protein sequence alignment. Bioinformatics. 2001; 17:700-712.

32. Kyte J, Doolittle RF. A simple method for displaying the hydropathic character of a protein. J Mol Biol. 1982; 157:105-132. 